\title{
Beyond Alternating Permutations: Pattern Avoidance in Young Diagrams and Tableaux
}

\author{
Nihal Gowravaram \\ Acton-Boxborough Regional High School \\ Acton, Massachusetts, U.S.A. \\ nihal1729@yahoo.com
}

\author{
Ravi Jagadeesan \\ Phillips Exeter Academy \\ Exeter, New Hampshire, U.S.A. \\ ravi.jagadeesan@gmail.com
}

Submitted: Mar 27, 2013; Accepted: Nov 5, 2013; Published: Nov 15, 2013

Mathematics Subject Classifications: 05A05; 05A19

\begin{abstract}
We investigate pattern avoidance in alternating permutations and generalizations thereof. First, we study pattern avoidance in an alternating analogue of Young diagrams. In particular, we extend Babson-West's notion of shape-Wilf equivalence to apply to alternating permutations and so generalize results of Backelin-West-Xin and Ouchterlony to alternating permutations. Second, we study pattern avoidance in the more general context of permutations with restricted ascents and descents. We consider a question of Lewis regarding permutations that are the reading words of thickened staircase Young tableaux, that is, permutations that have $k-1$ ascents followed by a descent, followed by $k-1$ ascents, et cetera. We determine the relative sizes of the sets of pattern-avoiding $(k-1)$-ascent permutations in terms of the forbidden pattern. Furthermore, inequalities in the sizes of sets of pattern-avoiding permutations in this context arise from further extensions of shape-equivalence type enumerations. This paper is the first of a two-paper series presenting the work of $\mathrm{Be}$ yond alternating permutations: Pattern avoidance in Young diagrams and tableaux (arXiv:1301.6796v1). The second in the series is Ascent-descent Young diagrams and pattern avoidance in alternating permutations (by the second author, submitted).
\end{abstract}

Keywords: pattern avoidance; alternating permutations; descent type permutations; Wilf equivalence; shape-Wilf equivalence

\section{Introduction}

For a nonnegative integer $n$, let $[n]$ denote the set $\{1,2,3, \ldots, n\}$, and let $S_{n}$ denote the set of permutations of $[n]$. We treat a permutation $w \in S_{n}$ as a sequence $w_{1} w_{2} w_{3} \cdots w_{n}$ that contains every element of $[n]$ exactly once. A permutation $w$ is said to contain a 
permutation $q$ if there is a subsequence of $w$ that is order-isomorphic to $q$. For example, the subsequence 246 of 214536 shows that 214536 contains 123 , and the only permutations that avoid 21 are the identity permutations. If $w$ does not contain $q$, then $w$ is said to avoid $q$.

The theory of pattern avoidance in permutations has connections to computer science, algebraic combinatorics, algebraic geometry, and representation theory. The fundamental question is to determine the size of the set $S_{n}(q)$ of permutations of length $n$ that avoid $q$. The theory first arose in the study of stack-sortable permutations; for example, Knuth [8] showed that stack-sortable permutations are exactly those that avoid the pattern 231. Additionally, generalized stack-sortable permutations are characterized by the avoidance of longer patterns; for an exposition, see [4, Chapter 8]. MacDonald [12] demonstrated that vexillary permutations are characterized by 2143 -avoidance. Furthermore, Lakshmibai and Sandhya [9] proved that permutations that simultaneously avoid 3412 and 4231 index smooth Schubert varieties. Billey and Warrington [3] showed that an interesting class of Kazhdan-Lusztig polynomials are indexed by permutations that simultaneously avoid 321 and four longer patterns. Tenner [18] maintains a database of situations in which pattern avoidance arises. These applications motivate the study of permutations that avoid patterns of arbitrary length.

Herb Wilf asked the question of when two patterns are equally difficult to avoid. If patterns $p$ and $q$ are such that $\left|S_{n}(p)\right|=\left|S_{n}(q)\right|$ for all $n$, we say that $p$ and $q$ are Wilfequivalent. The first non-trivial result of this type is the remarkable fact that all patterns of length 3 are Wilf-equivalent. Simion and Schmidt [15] gave a particularly elegant bijective proof. The bijections in Section 2 can be viewed as generalizations of [15].

A permutation $w \in S_{n}$ is called alternating if $w_{1}<w_{2}>w_{3}<\cdots$ and reverse alternating if $w_{1}>w_{2}<w_{3}>\cdots$. Reverse alternating permutations can be transformed into alternating permutations (and vice versa) by the complementation map that sends a permutation $w=w_{1} w_{2} \cdots w_{n}$ to $w^{c}=\left(n+1-w_{1}\right)\left(n+1-w_{2}\right) \cdots\left(n+1-w_{n}\right)$. Pattern-avoiding alternating permutations were first studied by Mansour [13] and by Deutsch and Reifegerste (documented in [17, Problem $\left.h^{7}\right]$ ), who proved that the number of alternating permutations of a given length that avoid a pattern of length 3 is a Catalan number. The enumeration is particularly interesting in that the number of permutations of a given length that avoid a pattern of length 3 is also a Catalan number. This suggests that pattern-avoiding alternating permutations have interesting enumerative properties both independently and in relation to ordinary pattern avoidance. In this paper, we develop further connections between the pattern avoidance of ordinary and alternating permutations while also generalizing beyond alternating permutations.

Given a pattern $q$, let $A_{n}(q)$ (resp. $\left.A_{n}^{\prime}(q)\right)$ denote the set of alternating (resp. reverse alternating) permutations of length $n$ that avoid $q$. If $p$ and $q$ are such that $\left|A_{n}(p)\right|=$ $\left|A_{n}(q)\right|$ (resp. $\left|A_{n}^{\prime}(p)\right|=\left|A_{n}^{\prime}(q)\right|$ ) for all even $n$, we say that $p$ and $q$ are equivalent for even-length alternating (resp. reverse alternating) permutations and we write $p \underset{\text { even }}{\sim} q$

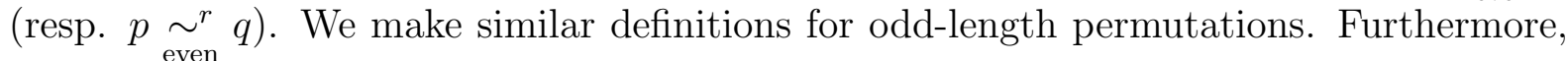
because $\left|A_{n}(q)\right|=\left|A_{n}^{\prime}\left(q^{c}\right)\right|$ for all $n, q$, the equivalence $p \underset{\text { even }}{\sim} q$ holds if and only if $p^{c} \underset{\text { even }}{\sim} q^{c}$, 


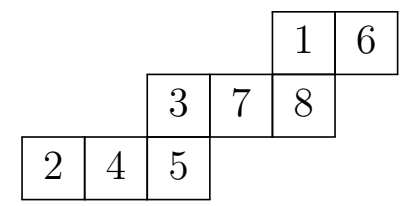

Figure 1: The permutation 24537816, which has descent type 3, is obtained by reading the entries of a skew standard Young tableau of shape $(6,5,3) /(4,2)$ from left to right and bottom to top. Because the final (top) row has only 2 entries, it is incomplete.

and similarly for the odd length equivalence.

We build on the work of Backelin, West, and Xin; their result is the following theorem.

Theorem $1.1([2]$, Theorem 2.1). For all $t \geqslant k$ and permutations $q$ of $\{k+1, k+2, \cdots, t\}$, the patterns $(k-1)(k-2)(k-3) \cdots 1 k q$ and $k(k-1)(k-2) \cdots 1 q$ are Wilf-equivalent.

Bóna [5] proved a variant of Theorem 1.1 for alternating permutations in the case of $k=2$ and $q=345 \cdots t$, while Ouchterlony [14] proved a similar result for doubly alternating permutations (alternating permutations whose inverse is alternating) in the case of $k=2$. In Section 2, we generalize the method of [1,2] to apply to permutations with restricted ascents and descents, using objects that we call AD-Young diagrams. This provides a framework of alternating shape-equivalence that attempts to fully extend Theorem 1.1 to alternating permutations. In Section 3, we use AD-Young diagrams to prove our main result Theorems 3.4, which is a variant of Theorem 1.1 for alternating permutations in the cases of $k=2$. We also consider patterns of short length.

A permutation $w$ is said to have descent type $k$ if

$$
w_{1}<w_{2}<\cdots<w_{k}>w_{k+1}<w_{k+2}<\cdots<w_{2 k}>w_{2 k+1}<w_{2 k+2}<\cdots
$$

Such a permutation may be thought of as a series of rows of length $k$ with values in strictly increasing order, with a possibly incomplete final row, as shown in Figure 1. Given pattern $q$, let $D_{n}^{k}(q)$ denote the set of permutations of descent type $k$ that avoid $q$. For example, alternating permutations have descent type 2 .

In [10], Lewis derived basic enumerations of pattern-avoiding descent type $k$ permutations. He computed the number of such permutations that avoid certain identity patterns. Lewis asked questions about descent type $k$ permutations and further generalizations of alternating permutation pattern avoidance in [11]. In Section 4, we study the relative sizes of $D_{n}^{k}(q)$ and $D_{n+1}^{k}(q)$ for fixed $k, q$. In Section 5, we apply the AD-Young diagram framework to generalizations of alternating permutations, and in Section 6 by posing open questions. In Section 7, we give the enumerative data that forms the basis for our conjectures.

This paper is the first of a two-paper series presenting the work of [6]; the second paper is [7] (by the second author, submitted). 


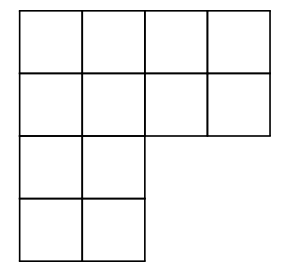

Figure 2: If $Y=\left(4^{2}, 2^{2}\right), A=\emptyset$, and $D=\{3\}$, then $(Y, A, D)$ is an AD-Young diagram.

\section{The AD-Young diagram framework}

Given a permutation $p$, let $M(p)$ denote its permutation matrix, and given matrices $A$ and $B$, let $A \oplus B=\left[\begin{array}{cc}A & 0 \\ 0 & B\end{array}\right]$. We assume that the reader is familiar with the basic terminology of Young diagrams and tableaux; see, for example, [4, Chapters 2 and 6]. We draw Young diagrams in English notation and use matrix coordinates, and for example $(1,2)$ is the second square in the first row of a Young diagram. Furthermore, we require all Young diagrams to have the same number of rows and columns.

In $[1,2]$, the notion of pattern avoidance is extended to transversals of a Young diagram, and analogue of the Wilf-equivalence of permutations is the shape-Wilf equivalence of permutation matrices. The critical theorem of [1] is that if $M$ and $N$ are shape-Wilf equivalent permutation matrices and $C$ is any permutation matrix, then the matrices $M \oplus C$ and $N \oplus C$ are shape-Wilf equivalent. We generalize the idea of a transversal of a Young diagram and refine shape-Wilf equivalence to apply to alternating permutations.

Definition 2.1. Let $Y$ be a Young diagram with $k$ rows. If $A$ and $D$ are disjoint subsets of $[k-1]$ such that if $i \in A \cup D$, then the $i$ th and $(i+1)$ st rows of $Y$ have the same length, then we call the triple $\mathcal{Y}=(Y, A, D)$ an $A D$-Young diagram. We call $Y$ the Young diagram of $\mathcal{Y}, A$ the required ascent set of $\mathcal{Y}$, and $D$ the required descent set of $\mathcal{Y}$. Figure 2 gives an example of an AD-Young diagram.

As in $[1,2,16]$, a transversal of Young diagram $Y$ is a set of squares $T=\left\{\left(i, t_{i}\right)\right\}$ such that every row and every column of $Y$ contains exactly one member of $T$.

Definition 2.2. Given a transversal $T=\left\{\left(i, t_{i}\right)\right\}$, let $\operatorname{Asc}(T)=\left\{i \in[k-1] \mid t_{i}<t_{i+1}\right\}$ and $\operatorname{Des}(T)=\left\{i \in[k-1] \mid t_{i}>t_{i+1}\right\}$. We call $\operatorname{Asc}(T)$ the ascent set of $T$ and $\operatorname{Des}(T)$ the descent set of $T$. If $A \subseteq A^{\prime}$ and $D \subseteq D^{\prime}$, then we say that $T$ a valid transversal of $\mathcal{Y}$.

Example 2.3. If $T$ is a transversal of a Young diagram $Y$, then $T$ is a valid transversal of the AD-Young diagram $(Y, \emptyset, \emptyset)$.

Except for a brief digression in Section 5, we restrict ourselves to the AD-Young analogues of alternating and reverse alternating permutations.

Definition 2.4. Given positive integers $x, y$ and an $\mathrm{AD}$-Young diagram $(Y, A, D)$ such that $Y$ has $k$ rows, we say that $(Y, A, D)$ is $x, y$-alternating if $A, D$ satisfy the property that if $x-1 \leqslant i \leqslant k-y$, then $i \in A$ if and only if $i+1 \in D$. 


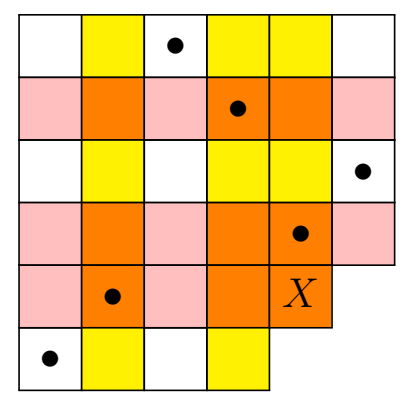

Figure 3: The transversal $T=\{(1,3),(2,4),(3,6),(4,5),(5,2),(6,1)\}$ of $Y=\left(6^{4}, 5,4\right)$ contains $M(231)$ because the restriction of $T$ to the yellow columns and the pink rows rows is a copy of $M(231)$ in $T$; we require that $X \in Y$. However, $T$ does not contain $M$ (4321); for example, the restriction of $T$ to rows $3,4,5,6$ and columns $1,2,5,6$ is not a copy of $M(4321)$ in $T$ because $(6,6) \notin Y$.

If $\mathcal{Y}$ is $x, y$-alternating, then $\mathcal{Y}$ is $a, b$-alternating for all $a, b$ with $a \geqslant x$ and $b \geqslant y$.

Definition 2.5. If $\mathcal{Y}$ is $1, y$-alternating, then we say that $\mathcal{Y}$ is $y$-alternating, while if $\mathcal{Y}$ is $2, y$-alternating, then we say that $\mathcal{Y}$ is $y$-semialternating.

Alternating AD-Young diagrams are the counterpart of alternating permutations, while semialternating AD-Young diagrams allow reverse alternating permutations.

Example 2.6. Let $Y=\left(4^{4}\right)$. Then, $(Y,\{1\},\{2\})$ is 1-alternating, while $(Y,\{1,3\},\{2\})$ is 2 -alternating but not 1-alternating. Furthermore, $(Y,\{2,4\},\{1,3\})$ is 1-semialternating but not $y$-alternating for $y \leqslant 4$.

The notion of pattern avoidance is exactly as in $[1,2,16]$; a transversal $T=\left\{\left(i, t_{i}\right)\right\}$ of a Young diagram $Y$ contains a $r \times r$ permutation matrix $M$ if there are rows $a_{1}<a_{2}<$ $\cdots<a_{r}$ and columns $b_{1}<b_{2}<\cdots<b_{r}$ of $Y$ such that $\left(a_{r}, b_{r}\right) \in Y$ and the restriction of $T$ to the rows $a_{i}$ and the columns $b_{i}$ has contains exactly the squares where $M$ has ones. If $T$ does not contain $M$, then $T$ avoids $M$ (see Figure 3). Given an AD-Young diagram $\mathcal{Y}$ and a permutation matrix $M$, let $S_{\mathcal{Y}}(M)$ denote the set of valid transversals of $\mathcal{Y}$ that avoid $M$.

Definition 2.7. If $M$ and $N$ are permutation matrices such that $\left|S_{\mathcal{Y}}(M)\right|=\left|S_{\mathcal{Y}}(N)\right|$ for all $x$-alternating AD-Young diagrams $\mathcal{Y}$, we say that $M$ and $N$ are shape-equivalent for $x$-alternating AD-Young diagrams; we write $M \underset{x-\mathrm{ASE}}{\sim} N$. If we have $\left|S_{\mathcal{Y}}(M)\right|=\left|S_{\mathcal{Y}}(N)\right|$ for all $x$-semialternating AD-Young diagrams $\mathcal{Y}$, then we say that $M$ and $N$ are shapeequivalent for x-semialternating AD-Young diagrams; we write $M \underset{x-\mathrm{SASE}}{\sim} N$.

If $M \underset{y-\mathrm{ASE}}{\sim} N$, then $M \underset{x-\mathrm{ASE}}{\sim} N$ for all positive integers $x \leqslant y$, while if $M \underset{y-\mathrm{SASE}}{\sim} N$, then $M \underset{x-\text { ASE }}{\sim} N$ and $M \underset{x-\text { SASE }}{\sim} N$ for all positive integers $x \leqslant y$. Because $(Y, \emptyset, \emptyset)$ is an alternating AD-Young diagram for every Young diagram $Y$, if $M$ and $N$ are shape-equivalent for 1-alternating AD-Young diagrams, then $M$ and $N$ are shape-Wilf equivalent; that is, 
for all Young diagrams $Y$, the number of transversals of $Y$ that avoid $M$ is the same as the number of transversals of $Y$ that avoid $N$. We explicitly connect alternating and semialternating AD-Young diagrams to alternating and reverse alternating permutations, respectively.

Proposition 2.8. Let $p$ and $q$ be permutations.

(a) If $M(p) \underset{1-A S E}{\sim} M(q)$, then $p \underset{\text { odd }}{\sim} q$.

(b) If $M(p) \underset{1-\widetilde{S A S E}}{\sim} M(q)$, then $p \underset{\text { even }}{\sim} \sim^{r}$.

(c) If $M(p) \underset{2-\widetilde{A S E}}{\sim} M(q)$, then $p \underset{\text { even }}{\sim} q$.

(d) If $M(p) \underset{2-\widetilde{S A S E}}{\sim} M(q)$, then $p \underset{\text { odd }}{\sim^{r}} q$.

Proof. We prove the first part; the remaining parts are similar. Fix a nonnegative integer $n$, and we will show that $\left|A_{2 n+1}(p)\right|=\left|A_{2 n+1}(q)\right|$. Consider the AD-Young diagram $\mathcal{Y}=$ $(Y, A, D)$ given by $Y=\left(2 n+1^{2 n+1}\right), A=\{1,3,5, \cdots, 2 n-1\}$, and $D=\{2,4,6, \cdots, 2 n\}$. It is clear that $\mathcal{Y}$ is 1 -alternating. Furthermore, a set $T=\left\{\left(i, b_{i}\right)\right\}$ is a valid transversal of $\mathcal{Y}$ if and only if $b=b_{1} b_{2} \cdots b_{2 n+1} \in A_{2 n+1}$, and $T$ avoids $M(p)$ if and only if $b$ avoids $p$. Hence, we have

$$
\left|A_{2 n+1}(p)\right|=\left|S_{\mathcal{Y}}(M(p))\right|=\left|S_{\mathcal{Y}}(M(q))\right|=\left|A_{2 n+1}(q)\right|,
$$

as desired.

\subsection{Generalization of Babson-West}

The extension of shape-equivalences from $M \sim N$ to $M \oplus C \sim N \oplus C$ is the analogue of [1, Theorems 1.6 and 1.9]. It is critical in generating infinite sets of nontrivial shapeequivalences. We have two variants, one for alternating AD-Young diagrams and one for semialternating AD-Young diagrams.

Theorem 2.9 (Extension Theorem). If permutation matrices $M$ and $M^{\prime}$ are shapeequivalent for $x$-alternating (resp. x-semialternating) $A D$-Young diagrams and $C$ is an $r \times r$ permutation matrix, then we have $M \oplus C \underset{(x+r)-A S E}{\sim} M^{\prime} \oplus C$ (resp. $\underset{(x+r)-S A S E}{\sim}$.

The remainder of this section will be devoted to the proof of Theorem 2.9. The first idea in the proof is to pass avoidance of $M \oplus C$ by a transversal of a large parent ADYoung diagram $\mathcal{Y}$ to avoidance of $M$ by a transversal of a smaller successor AD-Young diagram; this idea stems from the proof of [1, Theorems 1.6 and 1.9]. The successor map preserves the alternating property of AD-Young diagrams in the sense that if the parent is $(x+r)$-alternating and $C$ is an $r \times r$ matrix, then the successor is $x$-alternating. Furthermore, it sends valid transversals to valid transversals. 
The successor AD-Young diagram depends on the choice of transversal of $\mathcal{Y}$. However, similar to [1], we give an injection of the set of $M$-avoiding transversals of the successor diagram into the set of $M \oplus C$-avoiding transversals of $\mathcal{Y}$; the ascent set and the descent set of the successor diagram are chosen to facilitate this reinsertion procedure. We can then completely reduce the proof that $\left|S_{\mathcal{Y}}(M \oplus C)\right|=\left|S_{\mathcal{Y}}(N \oplus C)\right|$ to a statement about $M$-avoiding transversals of $x$-alternating AD-Young diagrams.

Fix an $r \times r$ permutation matrix $C$ and an $\mathrm{AD}$-Young diagram $\mathcal{Y}=(Y, A, D)(\mathcal{Y}$ need not be alternating). Let $T$ be a valid transversal of $\mathcal{Y}$. In the language of [1], call a square $(a, b) \in Y$ dominant with respect to $T$ if the restriction of $T$ to the region of squares $(x, y) \in Y$ with $x>a$ and $y>b$ contains $C$. Let $\mathcal{N}^{C}(T)$ denote the set of elements of $T$ that are not dominant with respect to $T$, and let $\mathcal{D}^{C}$ denote the family of sets $\mathcal{N}^{C}(T)$ as $T$ ranges over the valid transversals of $\mathcal{Y}$.

Lemma 2.10. The set of dominant squares of $\mathcal{Y}$ form a Young diagram. Furthermore, given the set $\mathcal{N}^{C}(T)$ and the permutation matrix $C$, one can recover the Young diagram of dominant squares.

Proof. See the proof of [1, Theorems 1.6 and 1.9].

Given a set $N=\mathcal{N}^{C}(T)$, let $d^{C}(N)$ denote the set of squares of $Y$ that are dominant with respect to $T$; the fact that $d$ is well-defined follows from Lemma 2.10. For a set $N$ of squares of $Y$ and a permutation matrix $P$, let $S_{\mathcal{Y}}^{N, C}(P)$ denote the set of valid transversals $T$ of $\mathcal{Y}$ that avoid the matrix $\left[\begin{array}{ll}P & 0 \\ 0 & C\end{array}\right]$ such that $\mathcal{N}^{C}(T)=N$. It is clear that we have

$$
\left|S_{\mathcal{Y}}\left(\left[\begin{array}{ll}
P & 0 \\
0 & C
\end{array}\right]\right)\right|=\sum_{N \in \mathcal{D}^{\mathcal{C}}}\left|S_{\mathcal{Y}}^{N}(P)\right| .
$$

We will define a function $f^{C}$ on $\mathcal{D}^{C}$ with the following key properties, to be proven after defining $f^{C}$. The value $f(N)$ is the successor diagram.

Lemma 2.11. For all $N \in \mathcal{D}^{C}, f^{C}(N)$ is an $A D$-Young diagram.

Lemma 2.12. If $\mathcal{Y}$ is $(x+r)$-alternating, then $f^{C}(N)$ is $x$-alternating.

Lemma 2.13. For all $N^{C} \in \mathcal{D}^{C}$ and all permutation matrices $P$, we have

$$
\left|S_{\mathcal{Y}}^{N}(P)\right|=\left|S_{f^{C}(N)}(P)\right|
$$

From the Young diagram of dominant squares $d\left(\mathcal{N}^{C}(T)\right)$, delete every row or column that contains a non-dominant square of $T$, and call the resulting Young diagram $Y^{\prime}$. Each row and column of $Y^{\prime}$ contains exactly 1 dominant member of $T$, and thus $Y^{\prime}$ has the same number of rows and columns. Suppose that $Y^{\prime}$ has $k$ rows, and that for all $1 \leqslant i \leqslant k$ the $i$ th column of $Y^{\prime}$ was the $c_{i}$ th column of $Y$ before the row and column deletion; similarly, suppose that for all $1 \leqslant i \leqslant k$, the $i$ th row of $Y^{\prime}$ was the $r_{i}$ th row of $Y$. Let

$$
A^{\prime}=\left\{i \in[k-1] \mid r_{i} \in A \text { and } r_{i+1}=r_{i}+1\right\},
$$




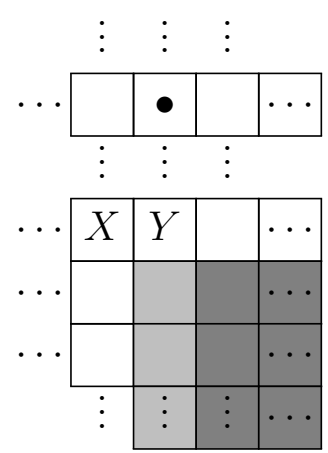

Figure 4: If the bullet point is an element of $T$, then there are no elements of $T$ among the light gray squares. Thus, if square $X$ is dominant, there must be a copy of $C$ among the dark gray squares, which implies that $Y$ is dominant.

and let

$$
D^{\prime}=\left\{i \in[k-1] \mid r_{i} \in D \text { and } r_{i+1}=r_{i}+1\right\} .
$$

By construction, the triple $\left(Y^{\prime}, A^{\prime}, D^{\prime}\right)$ depends only on $\mathcal{N}^{C}(T)$, and the set of dominant squares $d^{C}\left(\mathcal{N}^{C}(T)\right)$. Given a set $N=d^{C}\left(\mathcal{N}^{C}(T) \in \mathcal{D}\right.$, let $f^{C}(N)$ denote the corresponding triple $\left(Y^{\prime}, A^{\prime}, D^{\prime}\right)$. We prove that $f^{C}$ has the desired properties. Lemmata 2.11 and 2.12 will be immediate from the following lemma and proposition.

Lemma 2.14. Let $T=\left\{\left(i, b_{i}\right)\right\}$ be a valid transversal of $\mathcal{Y}$. If the square $(j, y)$ is dominant with respect to $T$ and $b_{j+1} \leqslant j$, then the square $(j+1, y)$ is dominant with respect to $T$.

Proof. See Figure 4. By the definition of dominant squares and because $(j, y)$ is dominant, there are rows $j<e_{1}<e_{2}<\cdots<e_{r}$ and columns $y<f_{1}<f_{2}<\cdots<f_{r}$ such that the restriction of $Y$ to the rows $e_{i}$ and the columns $f_{k}$ has members of $T$ exactly where $C$ has ones. If $j+1<e_{1}$, then the rows $e_{i}$ and the columns $f_{k}$ demonstrate that $(j+1, y)$ is dominant. Otherwise, we have $j+1 \geqslant e_{1}$, which implies that $e_{1}=j+1$. The only element of $T$ in row $j+1$ is $\left(j+1, b_{j+1}\right)$, and it follows that $b_{j+1}=f_{k}$ for some $k$. Regardless of $k$, we have $b_{j+1} \geqslant f_{1}>y$, which implies that $(j+1, y)$ is dominant by Lemma 2.10 .

Proposition 2.15. Let $N \in \mathcal{D}^{C}$ and let $f^{C}(N)=\left(Y^{\prime}, A^{\prime}, D^{\prime}\right)$. If $i \in A^{\prime}$ satisfies $r_{i}+1 \in$ $D$, then $r_{i}+1 \in D^{\prime}$. If $i \in D^{\prime}$ satisfies $r_{i}-1 \in A$, then $r_{i}-1 \in A^{\prime}$.

Proof. Let $T=\left\{i, b_{i}\right\}$ be a valid transversal of $\mathcal{Y}$ with $\mathcal{N}^{C}(T)=N$. If $i \in A^{\prime}$ satisfies $r_{i} \in D$, then $\left(r_{i}, b_{r_{i}}\right)$ is dominant and $b_{r_{i}+1}<b_{r_{i}}$. By Lemma 2.14, $\left(r_{i}+1, b_{r_{i}+1}\right)$ is dominant, and it follows that $r_{i+1}=r_{i}+1$ and $i+1 \in D^{\prime}$.

To prove the second part, we first prove that $\left(b_{r_{i}-1}, r_{i}-1\right)$ is dominant. Because $r_{i}-1 \in A$, we have $b_{r_{i}-1}<b_{r_{i}}$. Lemma 2.10 implies that $\left(r_{i}-1, b_{r_{i}-1}\right)$ is dominant. This yields that $r_{i-1}=r_{i}-1$, and the fact that $i-1 \in A^{\prime}$ follows by the definition of $A^{\prime}$.

Proof of Lemma 2.11. Let $f^{C}(N)=\left(Y^{\prime}, A^{\prime}, D^{\prime}\right)$. By construction and Lemma 2.10, $Y^{\prime}$ is a Young diagram. Because $A$ and $D$ are disjoint, the sets $A^{\prime}$ and $D^{\prime}$ are disjoint. Let $T=\left\{\left(i, b_{i}\right)\right\}$ be a valid transversal of $\mathcal{Y}$ with $\mathcal{N}^{C}(T)=N$, and suppose that $j \in A^{\prime} \cup D^{\prime}$. 


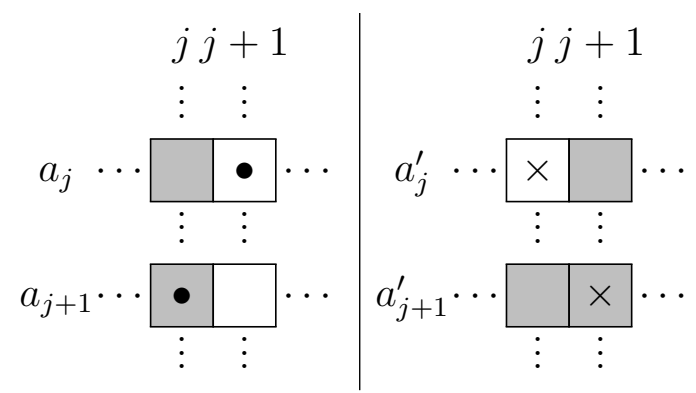

Figure 5: In the proof of Lemma 2.13, we show that if $j \in A$, then $j \in \operatorname{Asc}\left(h_{2}(T)\right)$. In all cases, the gray squares are dominant with respect to $T$, bulleted squares are elements of $h_{2}(T)$, and squares marked with a cross are elements of $T^{\prime}$. The left figure depicts Case 2: by Lemma 2.14, if the left bulleted square is dominant, then the right bulleted square must be as well. The right figure depicts Case 3: if the right crossed square is dominant, then the left crossed square must be as well by Lemma 2.10.

Let $y$ be the length of the $r_{j}$ th row of $d(N)$. By Lemma 2.10 and because $\left(r_{j}+1, b_{r_{j}+1}\right)$ is dominant, we have that $y \geqslant b_{r_{j}+1}$. Lemma 2.14 yields that $\left(r_{j}+1, y\right)$ is dominant, and thus the $r_{j}$ th and $\left(r_{j}+1\right)$ st rows of $d^{C}(N)$ have the same length. It follows that the $j$ th and $(j+1)$ st rows of $Y^{\prime}$ have the same length, as desired.

Proof of Lemma 2.12. Let $f^{C}(N)=\left(Y^{\prime}, A^{\prime}, D^{\prime}\right)$ and suppose that $Y^{\prime}$ has $k$ rows. Let $i \in D^{\prime}$ with $i \leqslant k-x$, and we prove that $i-1 \in A^{\prime}$. We have $r_{i} \leqslant r_{k-x} \leqslant r_{k}-x \leqslant n-r-x$. Because $\mathcal{Y}$ is $x+r$-alternating, this implies that that $r_{i}-1 \in A$. Proposition 2.15 yields that $i-1 \in A^{\prime}$, as desired. The proof that $i \in A^{\prime}$ with $i \leqslant k-x$ implies $i+1 \in D^{\prime}$ is similar.

Proof of Lemma 2.13. We prove the equality by establishing a bijection. Define the function $h: S_{\mathcal{Y}}^{N, C}(P) \rightarrow S_{f^{C}(N)}(P)$ by mapping a transversal $T \in S_{\mathcal{Y}}^{N, C}(P)$ to the image of $T$ after deleting any row or column that contains a non-dominant member of $T$. By definition of $N$, it is clear that $h(T)$ is a valid transversal of $f^{C}(N)$. Furthermore, if $h(T)$ contains $P$, then the set of dominant squares of $T$ contain $P$, which implies that $T$ contains $\left[\begin{array}{ll}P & 0 \\ 0 & C\end{array}\right]$. Hence, we can conclude that if $T \in S_{\mathcal{Y}}^{N, C}(P)$, then $h(T) \in S_{f^{C}(N)}(P)$. To show that $h$ is a bijection, we will show that it has an inverse. Consider the function $h_{2}: S_{f^{C}(N)}(P) \rightarrow S_{\mathcal{Y}}^{N, C}(P)$ given by mapping a valid transversal $T=\left\{\left(i, b_{i}\right)\right\} \in S_{f^{C}(N)}(P)$ to the transversal $T^{\prime}=N \cup\left\{\left(r_{i}, c_{b_{i}}\right) \mid\left(i, b_{i}\right) \in T\right\}$ of $Y$ (à priori, $h_{2}(T)$ is not necessarily an element of $\left.S_{\mathcal{Y}}^{N, C}(P)\right)$.

We claim that if $T \in S_{f^{C}(N)}(P)$, then $h_{2}(T)$ is a valid transversal of $\mathcal{Y}$. Let $T_{1}$ be a valid transversal of $\mathcal{Y}$ such that $\mathcal{N}^{C}\left(T_{1}\right)=N$; we introduce $T_{1}$ in order to exploit the fact that $N \in \mathcal{D}^{C}$. Let $h_{2}(T)=\left\{\left(i, a_{i}\right)\right\}$, and let $T_{1}=\left\{\left(i, a_{i}^{\prime}\right)\right\}$. Suppose that $j \in A$ and we will do casework on which of $j, j+1$ are among the rows $r_{i}$ to prove that $j$ is in the ascent set of $h_{2}(T)$. Figure 5 shows the casework geometrically. 
Case 1. Neither $j$ nor $j+1$ are among the indices $r_{i}$. Then, we have $a_{j}=a_{j}^{\prime}<a_{j+1}^{\prime}=$ $a_{j+1}$, as desired.

Case 2. $j$ is among the indices $r_{i}$ but $j+1$ is not. Assume for sake of contradiction that $a_{j}>a_{j+1}=a_{j+1}^{\prime}$. Because $\left(j, a_{j}\right)$ is dominant with respect to $T_{1}$, by Lemma 2.14 the square $\left(j+1, a_{j}\right)$ is dominant with respect to $T_{1}$, which implies that $\left(j+1, a_{j+1}^{\prime}\right)$ is dominant with respect to $T_{1}$ by Lemma 2.10. This contradicts the fact that $j+1$ is not among the rows $r_{i}$.

Case 3. $j+1$ is among the indices $r_{i}$ but $j$ is not. We claim that this is impossible. Because $a_{j}^{\prime}<a_{j+1}^{\prime}$ and $j+1$ is among the rows $r_{i}$, the square $\left(j, a_{j}^{\prime}\right)$ is dominant, which implies that $j$ is among the rows $r_{i}$.

Case 4. Both $j$ and $j+1$ are among the indices $r_{i}$. Suppose that $r_{x}=j$; then $x \in A^{\prime}$, which implies that $b_{x}<b_{x+1}$. Therefore, we have $a_{j}=c_{b_{x}}<c_{b_{x+1}}=a_{j+1}$, as desired.

The casework proves that $j$ is in the ascent set of $h^{\prime}(T)$. Suppose that $j \in D$, and we will prove that $j$ is in the descent set of $h_{2}(T)$ by dividing into the same cases.

Case 1. Neither $j$ nor $j+1$ are among the indices $r_{i}$. Then, we have $a_{j}=a_{j}^{\prime}>a_{j+1}^{\prime}=$ $a_{j+1}$, as desired.

Case 2. $j$ is among the indices $r_{i}$ but $j+1$ is not. Because $a_{j}^{\prime}>a_{j+1}^{\prime}$, by Lemma 2.14 the square $\left(j+1, a_{j}^{\prime}\right)$ is dominant with respect to $T_{1}$. This implies that $\left(j+1, a_{j+1}^{\prime}\right)$ by Lemma 2.10, which implies that $j+1$ is among the rows $r_{i}$.

Case 3. $j+1$ is among the indices $r_{i}$ but $j$ is not. By Lemma 2.10 and because $\left(j, a_{j}^{\prime}\right)$ is not dominant with respect to $T_{1}$, we have $a_{j}=a_{j}^{\prime}>a_{j+1}$, as desired.

Case 4. Both $j$ and $j+1$ are among the indices $r_{i}$. Suppose that $r_{x}=j$; then, we have $x \in D^{\prime}$, which implies that $b_{x}>b_{x+1}$. Therefore, we have $a_{j}=c_{b_{x}}>c_{b_{x+1}}=a_{j+1}$, as desired.

The casework establishes that every element of $D$ is in the descent set of $h^{\prime}(T)$, and it follows that $h_{2}(T)$ is a valid transversal of $\mathcal{Y}$. Because $T$ avoids $P$ and by the definition of dominant squares, $h_{2}(T)$ avoids $\left[\begin{array}{ll}P & 0 \\ 0 & C\end{array}\right]$. It is clear that $\mathcal{N}^{C}\left(h^{\prime}(T)\right)=N$, and this implies that $h_{2}(T) \in S_{\mathcal{Y}}^{N, C}(P)$ for all $T \in S_{f^{C}(N)}(P)$. Hence, $h$ and $h_{2}$ are inverses, and thus $h$ is a bijection. The lemma follows.

The following proposition is immediate from Lemma 2.13 and Equation 1, and we use it to prove Theorem 2.9 in the alternating case.

Proposition 2.16. For all permutation matrices $P, C$, we have

$$
\left|S_{\mathcal{Y}}\left(\left[\begin{array}{ll}
P & 0 \\
0 & C
\end{array}\right]\right)\right|=\sum_{N \in \mathcal{D}^{C}}\left|S_{f^{C}(N)}(P)\right| .
$$


Proof of Theorem 2.9 in the alternating case. Let $\mathcal{Y}$ be an $(x+r)$-alternating AD-Young diagram. By Lemma 2.12 and because $M \underset{x-\text { ASE }}{\sim} M^{\prime}$, we have $\left|S_{f^{C}(N)}(M)\right|=\left|S_{f^{C}(N)}\left(M^{\prime}\right)\right|$ for all $N \in \mathcal{D}^{C}$. Proposition 2.16 applied to $P=M$ and $P=M^{\prime}$ then yields that

$$
\left|S_{\mathcal{Y}}\left(\left[\begin{array}{cc}
M & 0 \\
0 & C
\end{array}\right]\right)\right|=\sum_{N \in \mathcal{D}^{C}}\left|S_{f^{C}(N)}(M)\right|=\sum_{N \in \mathcal{D}^{C}}\left|S_{f^{C}(N)}\left(M^{\prime}\right)\right|=\left|S_{\mathcal{Y}}\left(\left[\begin{array}{cc}
M^{\prime} & 0 \\
0 & C
\end{array}\right]\right)\right|,
$$

as desired.

In fact, the alternating $\mathrm{AD}$-Young diagrams arose as an attempt to provide a neat description for a superset of the closure of the set of AD-Young diagrams of the form $(Y, A, D)$ with $Y$ an $n \times n$ square, $A=[n-1] \cap(2 \mathbb{Z}+1)$, and $D=[n-1] \cap 2 \mathbb{Z}$ under such a successor map. The need to account for required ascents and descents significantly complicates both the definition of the successor map and the resulting proof of the Extension Theorem 2.9.

The proof of Theorem 2.9 in the semialternating case is almost identical. We simply replace Lemma 2.12 by the following lemma.

Lemma 2.17. If $\mathcal{Y}$ is $(x+r)$-semialternating and $N \in \mathcal{D}$, then the AD-Young diagram $f^{C}(N)$ is x-semialternating.

Proof. Let $f^{C}(N)=\left(Y^{\prime}, A^{\prime}, D^{\prime}\right)$ and suppose that $Y^{\prime}$ has $k$ rows. Let $i \in D^{\prime}$ with $1<i \leqslant k-x$, and we prove that $i-1 \in A^{\prime}$. We have $1<r_{i} \leqslant r_{k-x} \leqslant r_{k}-x \leqslant n-r-x$. Because $\mathcal{Y}$ is $x+r$-alternating, this implies that that $r_{i}-1 \in A$. Proposition 2.15 yields that $i-1 \in A^{\prime}$, as desired. The proof that $i \in A^{\prime}$ with $1 \leqslant i \leqslant k-x$ implies $i+1 \in D^{\prime}$ is similar.

\section{Shape-equivalences for AD-Young diagrams}

We now prove two shape-equivalences. For all positive integers $r$, let $I_{r}=M(123 \cdots r)$ and let $J_{r}=M(r(r-1)(r-2) \cdots 1)$. We will prove that $I_{2} \underset{1-\widetilde{A S E}_{2}}{\sim} J_{2}$ and $J_{3} \underset{1-\text { SASE }}{\sim} F_{3}$. Using the Extension Theorem 2.9, we will obtain infinitely many pairs of patterns that are equivalent for alternating and reverse alternating permutations in Theorems 3.4 and 3.6.

\subsection{The matrices $M(12)$ and $M(21)$ are shape-equivalent}

We will prove that $I_{2} \underset{1-\mathrm{ASE}}{\sim} J_{2}$; this will be the analogue of [1, Lemma 1.11], which proves that $I_{2}$ an $J_{2}$ are shape-Wilf equivalent. First, we prove an explicit enumeration of $S_{\mathcal{Y}}\left(I_{2}\right)$ and $S_{\mathcal{Y}}\left(J_{2}\right)$.

Proposition 3.1. For all $A D$-Young diagrams $\mathcal{Y}=(Y, A, D)$ such that $Y$ has $n$ rows, we have

$$
\left|S_{\mathcal{Y}}\left(I_{2}\right)\right|= \begin{cases}1 & \text { if } Y \supseteq(n, n-1, n-2, \ldots, 1) \text { and } A=\emptyset \\ 0 & \text { otherwise }\end{cases}
$$




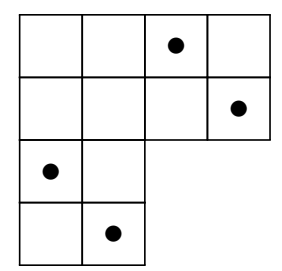

Figure 6: Suppose that $Y=\left(4^{2}, 2^{2}\right)$. In the fourth column, we select $(2,4)$ for $T$; then, we select $(1,3),(4,2),(3,1)$ in that order.

and

$$
\left|S_{\mathcal{Y}}\left(J_{2}\right)\right|= \begin{cases}1 & \text { if } Y \supseteq(n, n-1, n-2, \ldots, 1) \text { and } D=\emptyset \\ 0 & \text { otherwise. }\end{cases}
$$

The analogous result for ordinary Young diagrams is in the proof of [1, Lemma 1.11].

Proof. It is shown in the proof of [1, Lemma 1.11], that if $Y \nsupseteq(n, n-1, n-2, \ldots, 1)$, then $Y$ has no valid transversals, which implies that $\left|S_{\mathcal{Y}}\left(I_{2}\right)\right|=\left|S_{\mathcal{Y}}\left(J_{2}\right)\right|=0$.

To prove the first part, suppose that $x \in A$, and suppose that $T=\left\{\left(i, t_{i}\right)\right\}$ is a valid transversal of $Y$. Then, we have $t_{x}<t_{x+1}$ and the $x$ th and $x+1$ st rows of $Y$ have the same length. The restriction of $T$ to the $x$ th and $(x+1)$ st rows and the $b_{x}$ th and $b_{x+1}$ th columns of $Y$ demonstrates that $T$ contains $I_{2}$. Let $Y$ have $k$ rows. If $A=\emptyset$, then as in the proof of [1, Lemma 1.11], $T=\{(1, n),(2, n-1), \ldots,(n, 1)\}$ is the only element of $S_{\mathcal{Y}}\left(I_{2}\right)$.

To prove the second part, suppose that $x \in D$, and suppose that $T=\left\{\left(i, t_{i}\right)\right\}$ is a valid transversal of $Y$. Because the $x$ th and $(x+1)$ st rows of $Y$ have the same length, $T$ contains $J_{2}$. Suppose that $D=\emptyset$, and as in the proof of [1, Lemma 1.11], let $T=\left\{\left(i, b_{i}\right)\right\}$ be the transversal obtained by moving from the right column to the left column; for column $y$, select for $T$ a square in the lowest unoccupied row with at least $y$ squares. See Figure 6 for an example. Babson-West, in the proof of [1, Lemma 1.11], prove that this process returns the unique transversal of $Y$ that avoids $J_{2}$. We prove that it is a valid transversal of $\mathcal{Y}$. If $x \in A$, then the $x$ th and $(x+1)$ st rows of $Y$ have the same length, and let $m=\max \left\{b_{x}, b_{x+1}\right\}$. When we selected a square for the $m$ th column of $Y$, the $x+1$ st row of $Y$ was unoccupied, and by definition it has at least $m$ squares. Thus, we have $b_{x+1}=m$ and $x$ is an ascent of $T$. The fact that $T$ is a valid transversal of $\mathcal{Y}$ follows.

The following lemma is immediate from Proposition 3.1, and the subsequent theorem follows easily from Proposition 2.8, the Extension Theorem 2.9, and Lemma 3.2.

Lemma 3.2. We have that $I_{2} \underset{1-A S E}{\sim} J_{2}$.

Remark 3.3. In Definition 2.1, we require that if $i \in D$, then the $i$ th and $(i+1)$ st rows of $Y$ to have the same length in order for $(Y, A, D)$ to be an AD-Young diagram. For the necessity of this condition, consider the AD-Young diagram $\mathcal{Y}^{\prime}$ given by $Y^{\prime}=\left(3^{2}, 1\right)$, $A=\{1\}, D=\{2\}$. We have $\left|S_{\mathcal{Y}^{\prime}}(M(12))\right|=0$ but $\left|S_{\mathcal{Y}^{\prime}}(M(21))\right|=1$. 
Theorem 3.4. For all $t>2$ and all permutations $q$ of $[t] \backslash[2]$, the patterns $12 q$ and $21 q$ are equivalent for even- and odd-length alternating permutations.

Remark 3.5. An alternate proof of Theorem 3.4 via an isomorphism of generating trees is possible; see $[11,19]$ for an exposition of generating trees. However, such an isomorphism does not exist in the case of Theorem 3.6, even in the alternating case.

\subsection{The matrices $M(213)$ and $M(321)$ are shape-equivalent}

For a positive integer $r$, let $F_{r}$ denote the permutation matrix $M((r-1)(r-2) \cdots 1 r)$. In our recent paper [7], we prove that $F_{3} \underset{1-\mathrm{SASE}}{\sim} J_{3}$. Then, Proposition 2.8 and the Extension Theorem 2.9 imply the following theorem.

Theorem 3.6 ([7], Theorem 4.2). For all $t>3$ and all permutations $q$ of $[t] \backslash[3]$, the patterns $213 q$, and $321 q$ are equivalent for even- and odd-length reverse alternating permutations. The patterns $123 q, 213 q$, and $321 q$ are equivalent for even- and odd-length alternating permutations.

Taking complements in the statement of Theorem 3.6 for reverse alternating permutations yields the following corollary.

Corollary 3.7 ([7], Corollary 4.3). For all $t \geqslant 3$ and all permutations $q$ of $[t]$, the patterns $(t-1) t(t-2) q$ and $(t-2)(t-1)$ tq are equivalent for even- and odd-length alternating permutations.

\subsection{Applications of shape-equivalence to equivalences of short patterns}

The reverse of a permutation $w=w_{1} w_{2} \cdots w_{n}$ is the permutation $w^{r}=w_{n} w_{n-1} \cdots w_{1}$. Because reversal is an involution on odd-length alternating permutations, we have that $w \underset{\text { odd }}{\sim} w^{r}$ for all $w$ and likewise $w \underset{\text { even }}{\sim} w^{r c}$. Such equivalences are called trivial equivalences. We consider non-trivial equivalences among patterns of length 4 and 5 .

By Theorem 3.6, we have $1234 \underset{\text { odd }}{\sim} 2134 \underset{\text { odd }}{\sim} 3214$. By Theorem 3.4, we have 2143 odd 1243, which by reversal is equivalent to 3421, which is in turn equivalent to 2341 by Corollary 3.7. These equivalences constitute all possible equivalences for odd-length alternating permutations among patterns of length 4 due to the data of [11], thereby rederiving results of $[11,20]$. Similar logic yields that $1234 \underset{\text { even }}{\sim} 3214 \underset{\text { even }}{\sim} 2134 \underset{\text { even }}{\sim} 2143$ and $2341 \underset{\text { even }}{\sim} 3421$, which recovers results of $[5,11,20]$.

For patterns of length 5, we settled all possible equivalences except for 23451 od $34521,43215 \underset{\text { odd }}{\sim} 32145$, and $32145 \underset{\text { even }}{\sim} 43215 \underset{\text { even }}{\sim} 23451 \underset{\text { even }}{\sim} 34521$; this is 9 out of 11 possible equivalences for odd-length alternating permutations and 9 out of 12 possible equivalences for even-length alternating permutations. Except for 12345 odd 21345 and $12345 \underset{\text { even }}{\sim} 21345$, which are proven in [5], the equivalences among patterns of length 5 are 
new. Brute-force enumerations that describe all possible nontrivial equivalences among length 5 patterns are given in [11].

For patterns of length 6 , we described all possible nontrivial equivalences for both oddlength and even-length alternating permutations by brute-force enumeration. We present the list of possible equivalences in Section 7. Theorems 3.4 and 3.6 imply 35 out of 39 possible nontrivial equivalences for odd-length alternating permutations among patterns of length 6 , and 35 out of 45 possible nontrivial equivalences for even-length alternating permutations. Combinatorial blowup precludes the thorough examination of equivalences between patterns of length 7 .

\section{Generalized alternating permutations}

Throughout this section let $p=p_{1} p_{2} \cdots p_{n}$ be a permutation of length $n$. Similarly, let $q=q_{1} q_{2} q_{3} \cdots q_{b}$ be a pattern of length $b$. In [11], an operation called extension was used to recursively generate pattern-avoiding permutations of length $n+1$ from such permutations of length $n$. The procedure itself involved appending a new value to the end of a permutation. However, in the context of permutation of descent type $k$, this procedure restricts us to only extending values $v \geqslant p_{n}$. We require more flexibility in choosing which values to add, so we define a new method to add a value.

Definition 4.1. Let $p \in S_{n}$ be a permutation of descent type $k$ and let $v \in[n+1]$. Define $v \mapsto p$, the injection of $v$ into $p$ as follows: we first increment all values of $p$ that are greater than or equal to $v$ and then append $v$ to get a permutation $p^{\prime}$. Then if $p$ had an incomplete final row, we rearrange the elements of the final row of $p^{\prime}$ to be in increasing order. If $p$ had a complete final row and $v \leqslant p_{n}$, we simply define $v \mapsto p$ as $p^{\prime}$. However, if $v>p_{n}$, we swap the last two entries of $p^{\prime}$. If $w=v \mapsto p$ for some $v$, then we say that $w$ is a child of $v$ and $v$ is a parent of $w$.

Remark 4.2. Permutations need not have a unique parent.

Example 4.3. Consider the permutation 35624718 of descent type $k=3$. We have $4 \mapsto$ $35624718=367258149$, because $p^{\prime}=36725819$, and appending 4 to $p^{\prime}$ gives 367258194 . Rearranging the final row then yields 367258149.

We omit the proof that if $p$ has descent type $k$, then every child of $p$ also descent type $k$. It is clear, however, that every child of $p$ contains $p$, and therefore if a child of $p$ avoids a pattern $q$, then so does $p$.

In Section 4.1, the primary nontrivial result is that $\left|D_{n}^{k}(q)\right| \leqslant\left|D_{n+1}^{k}(q)\right|$ for all patterns $q$ except for the trivial counterexample of the identity permutation when $k \geqslant b$. Additionally, in Section 4.3, we investigate repetitive patterns, patterns which are characterized by pattern avoidance of a particular triplet of patterns. What is especially interesting about these patterns, as we show in Section 4.3, is that $\left|D_{n}^{k}(q)\right|=\left|D_{n+1}^{k}(q)\right|$ for particular values of $n$ and repetitive patterns $q$. In conjunction with this, for all non-repetitive patterns, in Section 4.2 we show that $\left|D_{n}^{k}(q)\right|<\left|D_{n+1}^{k}(q)\right|$. Since $D_{n}^{k}(q)$ is trivial to understand when $n<k$, as $\left|D_{n}^{k}(q)\right|=1$ (or 0 for short identity permutations), it shall be assumed 
throughout this section $n \geqslant k$. For similar reasons, since $q=12,21$ are trivial cases as well, we shall assume that $b \geqslant 3$.

\subsection{Nonstrict Case: $\left|D_{n}^{k}(q)\right| \leqslant\left|D_{n+1}^{k}(q)\right|$}

We shall show that $\left|D_{n}^{k}(q)\right| \leqslant\left|D_{n+1}^{k}(q)\right|$; this is fairly intuitive for as we consider longer length permutations, we would expect more permutations to avoid the fixed pattern. We prove the following theorem.

Theorem 4.4. Let $k \leqslant n$ be positive integers and let $q \notin\{21,1,12,123, \ldots, 123 \cdots k\}$. Then, there is an injection $f: D_{n}^{k}(q) \hookrightarrow D_{n+1}^{k}(q)$ such that for all $p, f(p)$ is a child of $p$. In particular, we have $\left|D_{n}^{k}(q)\right| \leqslant\left|D_{n+1}^{k}(q)\right|$.

In other words, for each parent, we are choosing a different child. In the case where $q=123 \cdots b$ with $b \leqslant k, k$ is so large that any sufficiently long permutation with descent type $k$ contains $q$ when $n \geqslant b$, since the first $k$ values of the permutation are in strictly increasing order.

Proof. Fix $q$ satisfying the theorem conditions.

Let $p \in D_{n}^{k}(q)$. Define a consecutive block to be a subset of consecutive cells that are consecutive in value as well; i. e. $a_{i}<a_{i+1}<\cdots<a_{j}$ for $i<i+1<\cdots<j$, and $a_{s}-a_{s-1}=1$ when $i<s \leqslant j$. We call the value $a_{j}$ the anchor of the consecutive block. We define the block function $B(q)$ to return the length of the consecutive block anchored at $q_{b}$. (Note that this function is only defined for patterns with $q_{b}=b$. ) Note that if the pattern is the identity pattern, then the function returns $b$. The following algorithm defines $f(p)$.

- If $q_{b}=b$ :

- If $n=k m+s, 0 \leqslant s<B(q)$, inject 1 .

- If $n=k m+s, B(q) \leqslant s<k$, inject $p_{n-B(q)+1}$.

- If $q_{b} \neq b$ :

$$
\begin{aligned}
& \text { - If } n=k m+s, 0<s<k, \text { inject } n+1 . \\
& \text { - If } n=k m: \\
& \begin{aligned}
* & \text { If } q_{b}=1: \\
& \cdot \text { If } q_{b-1}=2, \text { inject } n+1 . \\
& \cdot \text { If } q_{b-1} \neq 2, \text { inject } p_{k m} . \\
& * \text { If } q_{b} \neq 1, \text { inject } 1 .
\end{aligned}
\end{aligned}
$$

Example 4.5. Let $q=2134$. Then $B(q)=B(2134)=2$. Consider permutation $p=23514$ with $k=3$. Then, by the algorithm, the child permutation $p^{\prime}=f(p)=346125$ since we inject the value $p_{4}=1$. Similarly by the algorithm, $f\left(p^{\prime}\right)=4572361$ as we inject the value 1 into $p^{\prime}$. 
We now prove $f$ is injective via casework.

Case 1. We have $q_{b}=b$.

Subcase 1.1. We have $n=k m+s$ with $0 \leqslant s<B(q)$. We claim that the injection of the value 1 into the final row will result in a $p^{\prime} \in D_{n+1}^{k}(q)$. Since 1 is the smallest value in the permutation, it must be the first value in the final row. Since $n<k m+B(q)$, there exist at most $B(q)-1$ values to the right of 1 in $p^{\prime}$. However, by definition of $B(q)$, there are at least $B(q)$ values to the right of 1 in $q$. Thus, $p^{\prime}$ avoids $q$ as desired.

Subcase 1.2. We have $n=k m+s$ with $B(q) \leqslant s<k$. Let $p_{n-B(q)+1}=f$. We claim that in this subcase, the injection of the value $f$ into the final row will result in a valid $p^{\prime} \in D_{n+1}^{k}(q)$. Assume, for the sake of contradiction, $p^{\prime}$ contains $q$. As a result of the injection, $p_{n-B(q)+2}=f+1$. If only $p_{n-B(q)+1}$ is part of the subsequence, this is a contradiction because then the same subsequence is in $p$, indicating that $p$ contains $q$. Similarly, if only $p_{n-B(q)+2}$ is part of the subsequence, since $p_{n-B(q)+1}$ and $p_{n-B(q)+2}$ are consecutive integers, we can simply swap the corresponding position of $p_{n-B(q)+2}$ for $p_{n-B(q)+1}$, resulting in another contradiction. Therefore, both of $p_{n-B(q)+1}$ or $p_{n-B(q)+2}$ must be in the subsequence that is order-isomorphic to $q$. There remain two nontrivial cases to consider: if both $p_{n-B(q)+1}$ and $p_{n-B(q)+2}$ are isomorphic to values part of the consecutive block, and if both $p_{n-B(q)+1}$ and $p_{n-B(q)+2}$ are isomorphic to values not part of the consecutive block. If the former case were possible, then $B(q)>1$, implying that $p_{n-B(q)+3}$ exists. However since $q_{b}=b, p_{n-B(q)+2}$ is the largest value in the subsequence. But, then, we could substitute the $p_{n-B(q)+3}$ term for the $p_{n-B(q)+2}$, which is a contradiction since this then implies that the original $p$ contained $q$. The latter case leads to contradiction similarly.

Case 2. We have $q_{b} \neq b$.

Subcase 2.1. $k$ does not divide $m$. Suppose that $n=k m+s$ with $0<s<k$. In this subcase, the final row has at least one cell, but must still be incomplete (since $s<k$ ). Thus, there are no restrictions on what can be appended to the row. So, the algorithm is simply to inject $n+1$ (in this case, this is simply appending $n+1$ to the end). Clearly, since this must be the largest value in the permutation, and the value is in the last row, $p_{n+1}=n+1$. Thus, the $p^{\prime}$ that results must avoid $q$, since clearly $p_{n+1}$ cannot be part of a subsequence order-isomorphic to $q$ since $q_{b} \neq b$ and since the original permutation $p$ avoids $q$.

Subcase 2.2. $k$ divides $n$. Suppose that $n=k m$. This subcase is slightly more complicated. Here, we have an added restriction; by definition, $p_{k m}>p_{k m+1}$. We will proceed with further casework.

If $q_{b}=1$ and $q_{b-1}=2$, the algorithm is to simply inject $n+1$. As a result, $p_{k m}=n+1$, and then the old value of $p_{k m}$ is bumped up into the next row. 
This swapping is essential because $p_{k m}>p_{k m+1}$. Clearly, $p_{k m}$ cannot be part of any subsequence order-isomorphic to $q$, because the value of $n+1$ cannot correspond to the 1 nor 2 in $q$. Thus, since the original $p$ avoids $q$, and the relative positions of the values in $p^{\prime}$ are invariant from $p, p^{\prime}$ avoids $q$ as well, and so, $p^{\prime} \in D_{n+1}^{k}(q)$.

If $q_{b}=1$ and $q_{b-1} \neq 2$, the algorithm is to inject $f$ into the new row. Let $p_{k m}$ $=f$. So, upon the injection, $p_{k m}=f+1$ and $p_{k m}=p_{k m+1}+1$. We claim that the resulting $p^{\prime}$ avoids $q$. Assume, for the sake of contradiction, $p^{\prime}$ contains $q$ and so there exists some subsequence of $p^{\prime}$ that is order-isomorphic to $q$. Since $p$ avoids $q$, the only situations to consider are if only one of $p_{k m}$ and $p_{k m+1}$ are part of the subsequence, or if both $p_{k m}$ and $p_{k m+1}$ are in the subsequence. These situations are easily tractable, yielding contradictions in a manner similar to the proofs above. Thus, $p^{\prime}$ avoids $q$ as well, and so, $p^{\prime} \in D_{n+1}^{k}(q)$.

If $q_{b} \neq 1$, we simply inject 1 into the final row (i.e. $p_{k m+1}=1$ ). Clearly, then, since 1 is the smallest value in the permutation, $p_{k m+1}$ cannot be part of a subsequence that is order-isomorphic to $q$ since $q_{b} \neq 1$. Thus, since the original permutation $p$ avoided $q, p^{\prime}$ avoids $q$ as well, and so, $p^{\prime} \in D_{n+1}^{k}(q)$.

The above procedures are all reversible as we can easily undo the injection. The casework shows that the algorithm is indeed injective and that the children $f(p)$ are pairwise distinct.

\subsection{Strict Case: $\left|D_{n}^{k}(q)\right|<\left|D_{n+1}^{k}(q)\right|$}

Call a pattern repetitive if it avoids $321,132,231$. Similarly, a pattern is non-repetitive if it contains at least one of $321,132,231$. We prove the following theorem.

Theorem 4.6. For all non-repetitive patterns $q$ with $q \notin\{21,1,12, \ldots, 123 \cdots\}$ and all $k, n$, we have $\left|D_{n}^{k}(q)\right|<\left|D_{n+1}^{k}(q)\right|$. If $q$ is repetitive and $k \mid n$, then $\left|D_{n}^{k}(q)\right|<\left|D_{n+1}^{k}(q)\right|$ as well.

Our approach will be an inductive one. Lemma 4.7 captures the overall nature of induction from $\left|D_{n}^{k}(q)\right|$ to $\left|D_{n+1}^{k}(q)\right|$, while the rest of the section more specifically details our algorithm through casework based on the value of $n$.

The following lemma provides the framework for our inductive argument.

Lemma 4.7. Let $q$ and $q^{\prime}$ be two patterns such that $q$ contains $q^{\prime}$. If $\left|D_{n}^{k}\left(q^{\prime}\right)\right|<\left|D_{n+1}^{k}\left(q^{\prime}\right)\right|$, it must also be true that $\left|D_{n}^{k}(q)\right|<\left|D_{n+1}^{k}(q)\right|$.

Proof. The key idea is that any parent of a permutation that avoids $q^{\prime}$ also avoids $q^{\prime}$. Therefore, under the theorem conditions, the assignment of children of Theorem 4.4 must miss a permutation in $D_{n+1}^{k}\left(q^{\prime}\right)$. Let $f: D_{n}^{k}(q) \hookrightarrow D_{n+1}^{k}(q)$ be defined from Theorem 4.4. If $f(p) \in D_{n+1}^{k}(q)$, then a child of $p$ does not contain $q$, and therefore $p$ does contain $q$ and $p \in D_{n}^{k}(q)$. Because $\left|D_{n}^{k}\left(q^{\prime}\right)\right|<\left|D_{n+1}^{k}\left(q^{\prime}\right)\right|$, there is an element of $D_{n+1}^{k}\left(q^{\prime}\right)$ that is outside $f\left(D_{n}^{k}\left(q^{\prime}\right)\right) \supseteq D_{n+1}^{k}\left(q^{\prime}\right) \cap f\left(D_{n}^{k}(q)\right)$. Hence, $f$ is not surjective, and therefore $\left|D_{n}^{k}(q)\right|<\left|D_{n+1}^{k}(q)\right|$. 
Proof of Theorem 4.6. We now shall proceed with casework based on the value of $n$. We apply Lemma 4.7 for the desired result. We divide into cases based on the residue of $n$ on division by $k$. We first consider permutations that avoid at least one of 213, 312; then, we do casework to finish the argument.

Case 1. $k$ does not divide $n$. Suppose that $n=k m+s$ with $0<s<k$.

We consider non-repetitive patterns, and we revisit repetitive patterns in Case 2 . Let $q$ be a non-repetitive pattern, and we do casework on which of 132, 231, 321 that $q$ contains.

Subcase 1.1. $q$ contains 132 or 231. Much of this case has already been shown by Lewis in [10]. Recall that permutations of descent type $k$ are obtaining from reading skew Young tableaux of a particular shape (see Figure 1). Suppose that permutations of length $n$ of descent type $k$ are identified with tableaux of shape $\lambda / \mu$ and such permutations of length $n+1$ are identified with tableaux of shape $\lambda^{\prime} / \mu^{\prime}$. Let $r=\left\lceil\frac{n}{k}\right\rceil$; then $\lambda$ and $\lambda^{\prime}$ have $r$ rows. By [10, Corollary 7.3], there is a bijection between $D_{n}^{k}(132)$ and the set of Young diagrams $Y \subseteq$ $\left(\lambda_{1}-\lambda_{r}, \lambda_{2}-\lambda_{r}, \cdots, \lambda_{r-1}-\lambda_{r}\right)$, and similarly for $D_{n+1}^{k}(q)$ and $\lambda^{\prime}$. However, we have $\lambda_{1}^{\prime}=\lambda_{1}+1$ and $\lambda_{i}=\lambda_{i}^{\prime}$ for all $i>1$. Therefore, $\left(\lambda_{1}-\lambda_{r}, \lambda_{2}-\lambda_{r}, \cdots, \lambda_{r-1}-\right.$ $\left.\lambda_{r}\right) \subsetneq\left(\lambda_{1}^{\prime}-\lambda_{r}^{\prime}, \lambda_{2}^{\prime}-\lambda_{r}^{\prime}, \cdots, \lambda_{r-1}^{\prime}-\lambda_{r}^{\prime}\right)$ and the fact that $\left|D_{n}^{k}(q)<\right| D_{n+1}^{k}(q) \mid$ follows. A similar argument using [10, Corollary 7.6] settles the 231 case.

Subcase 1.2. $q$ contains 321. Consider a permutation $p$ in $\left|D_{n}^{k}(321)\right|$. Clearly, we may simply append the value $n+1$ to $p$ to obtain an element of $D_{n+1}^{k}(321)$. However, we can also replace $p_{k m}$ with the value $n+1$, and inject the $p_{k m}$ value into the final row. Since the sets of permutations derived from the two procedures are disjoint due to different locations of $n+1$, we have $\left|D_{n+1}^{k}(321)\right| \geqslant$ $2\left|D_{n}^{k}(321)\right|$.

Lemma 4.7 implies that, for all $k \nmid n$ and $q$ non-repetitive, we have $\left|D_{n}^{k}(q)\right|<$ $\left|D_{n+1}^{k}(q)\right|$.

Case 2. $k$ divides $n$. Suppose that $n=k m$. This case is slightly more complicated than the previous case. When $k=2$ (alternating permutations), we firstly consider patterns that contain at least one of 123, 213, and 312. However, when $k>2$, we instead consider patterns that contain at least one of 321, 213, and 312. For both of these triples of patterns, there are patterns that avoid all three, which we address at the end of the casework by considering all such patterns of length 4 . It is important to note that unlike the previous subsection, this case includes repetitive patterns as well.

Subcase 2.1. $q$ contains 123 .

Since we are only considering the $k=2$ case, we may simply inject the values 1 and 2 while preserving 123-avoidance. Since these injections result in distinct permutations, we have $\left|D_{2 m}^{2}(123)\right|<\left|D_{2 m+1}^{2}(123)\right|$. 


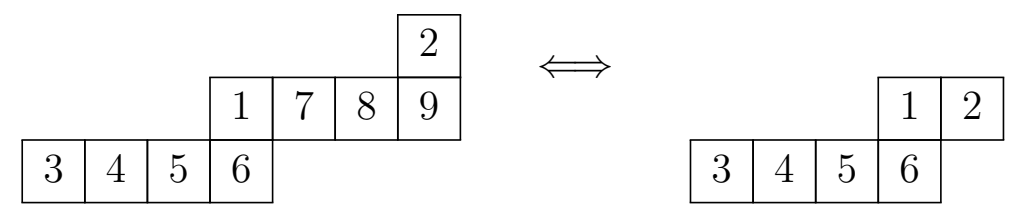

Figure 7: Removing the largest consecutive block $(7,8,9)$ from 345617892 and collapsing the final row into the row beneath it results in another valid permutation avoiding 321 . Reversing the deletion of a consecutive block is clear as well, for inserting $(7,8,9)$ into 345612 bumps up the final value 2 into a new row and the consecutive block fills the prior final row.

Subcase 2.2. $q$ contains 213 or 312 . The idea here is very similar to the preceding one. Since the permutation $p$ has descent type $k$, we may clearly inject any value $v \leqslant k$ into $p$. One may verify that this operation is reversible. Therefore, we have $\left|D_{k m+1}^{k}(q)\right| \geqslant k\left|D_{k m}^{k}(q)\right|>\left|D_{k m}^{k}(q)\right|$ for $q=213,312$.

Subcase 2.3. $q$ contains 321 . In this case we prove the following more interesting result.

Proposition 4.8. For all $k, m>1$, we have

$$
\left|D_{k m+1}^{k}(321)\right|=\sum_{i=k(m-1)+2}^{k m}\left|D_{i}^{k}(321)\right| .
$$

Proof Idea. The idea of this proof is to reintroduce the notion of the consecutive block. First, it is important to note that for $p \in D_{k m+1}^{k}(321), p_{k m}=k m+1$. Thus, a bijection is achieved by simply inserting a consecutive block into a permutation and in the other direction, removing the largest such block from a permutation. Structurally, a consecutive block is a group of consecutive cells of a permutation that are in the same row and also consecutively ascending in value. A graphical example of this bijection is depicted in Figure 7. The proposition follows from this bijection.

Note that $\left|D_{k m+1}^{k}(321)\right|=\left|D_{k m}^{k}(321)\right|$ when $k=2$; however, when $k>2$, we have $\left|D_{k m+1}^{k}(321)\right|>\left|D_{k m}^{k}(321)\right|$.

Subcase 2.4. $q$ avoids 123,213 , and 312. Consider the length-4 patterns that avoid 123, 213, and 312; they are 4321, 1432, 2431, and 3421. Note that every pattern that avoids 123, 213, and 312 must contain at least one of these 4 patterns. We prove that for each of these patterns there exists a second valid injection, distinct from the one provided in Section 4.1, for every $p \in D_{k m}^{k}(q)$.

- 4321 and 3421: Inject $n$ if $p_{k m} \neq n$. Inject $n-1$ if $p_{k m}=n$.

- 1432 and 2431: Inject $n+1$.

The verification that this algorithm proves that $\left|D_{k m}^{k}(q)\right|<\left|D_{k m+1}^{k}(q)\right|$ is straightforward. 
Subcase 2.5. $q$ avoids 321, 213, and 312. Consider the length- 4 patterns that avoid 321,213 , and 312 ; they are $1234,1243,1342$, and 2341 . Note that every pattern that avoids 321,213 , and 312 must contain at least one of these 4 patterns. As before, we simply provide the algorithm.

- 1234 and 1243: Inject 2.

- 1342: Inject $p_{k m}$.

- 2341: Inject $n-1$ if $p_{k m}=n$. Inject $p_{k m}+2$ if $p_{k m} \neq n$.

Combining all the cases and applying Lemma 4.7 yields that $\left|D_{n}^{k}(q)\right|<\left|D_{n+1}^{k}(q)\right|$ for all $q$ and $k \mid n$.

\subsection{Equality Case: $\left|D_{n}^{k}(q)\right|=\left|D_{n+1}^{k}(q)\right|$ (Repetitive Patterns)}

In the previous section, we defined repetitive patterns to be those that avoided 321, 132 and 231 simultaneously. Now, we determine the structure of such patterns. Fix the location of the 1 . Since the pattern simultaneouly avoids 231 and 321, there can be at most one value to the left of the 1 . Additionally, since the pattern avoids 132, all values to the right of the 1 must be in strictly increasing order. Thus, the pattern $q$ must be an identity pattern, or must be of the form $t 123 \cdots(t-1)(t+1) \cdots b$, where $q_{1}=t$ and $b$ is the length of the pattern.

We consider the case when $q$ is a non-identity, repetitive pattern. The sequence $\left\{D_{n}^{k}(q)\right\}$ has predictable repetitions among consecutive terms. We prove the following theorem.

Theorem 4.9. For all $k \geqslant b-1$ and all non-identity repetitive patterns $q$ of length $b$,

$$
\left|D_{k m+(b-2)}^{k}(q)\right|=\left|D_{k m+(b-1)}^{k}(q)\right|=\left|D_{k m+b}^{k}(q)\right|=\cdots=\left|D_{k m+k}^{k}(q)\right| .
$$

We divide the proof into two separate cases. Lemmata 4.10 and 4.11 tackle the case when $q_{1}=b$. Similarly, Lemmata 4.12 and 4.13 deal with the general case.

Lemma 4.10. For $q$ with $q_{1}=b$ and $b-1 \leqslant x \leqslant k$, and for all $p \in D_{k m+x}^{k}(q)$, we have $p_{k m+x}=k m+x$.

Proof. Assume for the sake of contradiction that $k m+x$ is not part of the final row in $p$. Then there exists an index $i<k m+1$ such that $p_{i}=k m+x$. Because $x \geqslant t-1$, we have $p_{k m+1}<p_{k m+2}<\cdots<p_{k m+(t-1)}$. However, because $i<k m+1$ and $p_{i}>$ $p_{k m+1}$, the pattern $p_{i} p_{k m+1} p_{k m+2} \cdots p_{k m+(b-1)}$ is order-isomorphic to $q$. Thus, $p$ contains $q$, contradiction. Consequently, $k m+x$ must be part of the final row in $p$. However, by definition, since each block is strictly ordered from least to greatest, and because $k m+x$ is the largest value in the block, we have $p_{k m+x}=k m+x$, as desired.

Lemma 4.11. For $q$ with $q_{1}=b$, all $b-2 \leqslant x \leqslant k-1$, we have $\left|D_{k m+x}^{k}(q)\right|=$ $\left|D_{k m+(x+1)}^{k}(q)\right|$. 


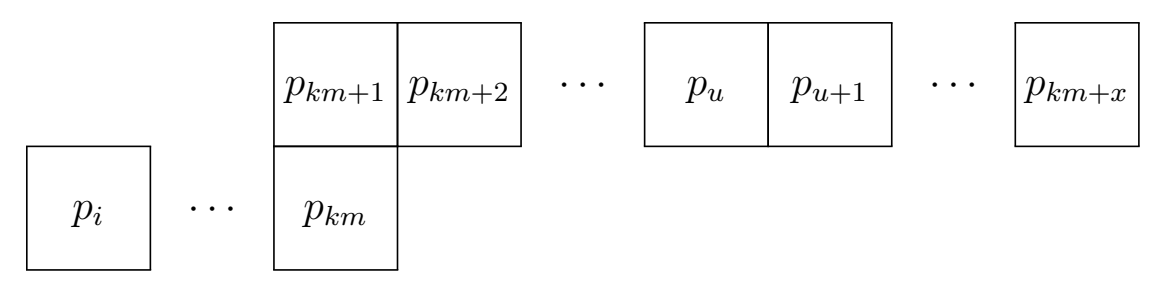

Figure 8: The proof of Lemma 4.12 is shown, where $u=k m+x+t-b$. If $p_{k m+x+t-b}<$ $p_{i}<p_{k m+x+t-b+1}$, then $p$ contains $q$, contradiction.

Proof. We exhibit a bijection to prove the lemma. First we describe a function from $D_{k m+x}^{k}(q)$ to $D_{k m+(x+1)}^{k}(q)$. For $p \in D^{k} k m+x(q)$, the injection of $p_{k m+x+1}=k m+x+1$ results in a $p^{\prime} \in D_{k m+(x+1)}^{k, l=0}(q)$, since this injection clearly maintains all original relations prior to the injection and is valid because then $p_{k m+(x+1)}$ holds the largest value in the permutation.

We now describe the inverse function from $D_{k m+(x+1)}^{k}(q)$ to $D_{k m+x}^{k}(q)$. By Lemma 4.10, for $s \in D_{k m+(x+1)}^{k}(q), s_{k m+(x+1)}=k m+(x+1)$. Thus, we may simply remove $s_{k m+(x+1)}$ from $s$ to get a permutation $s^{\prime} \in D_{k m+x}^{k}(q)$.

The two functions are clearly inverse. Therefore, they are bijective, and the lemma follows.

Now we tackle the more general case.

Lemma 4.12. For $q$ with $q_{1}=t \neq b$, all $b-1 \leqslant x \leqslant k$, and all $p$ in $D_{k m+x}^{k}(q)$, we have $p_{k m+(x+t-b+1)}=p_{k m+(x+t-b)}+1$.

Proof. Assume for the sake of contradiction that $p_{k m+(x+t-b)}$ and $p_{k m+(x+t-b+1)}$ are not consecutive values. There exists an index $i$ such that $p_{k m+(x+t-b)}<p_{i}<p_{k m+(x+t-b+1)}$. Clearly, $p_{i}$ is not in the final block of $p$ (it it was, its "cell" would be in between those of $p_{k m+(x+t-b)}$ and $p_{k m+(x+t-b+1)}$, which is impossible since $p_{k m+(x+t-b)}$ and $p_{k m+(x+t-b+1)}$ are adjacent cells) and therefore, $p_{i}$ is in an earlier block of $p$. Hence, $i<k m+1$ and $p_{i}>p_{k m+(x-b+2)}\left(\right.$ since $p_{k m+(x-b+2)}$ is in the final block, yet $\left.p_{k m+(x-b+2)} \leqslant p_{k m+(x+t-b)}\right)$. Figure 8 shows the argument geometrically.

However, since

$$
p_{k m+(x-b+2)}<p_{k m+(x-b+3)}<\cdots<p_{k m+(x+t-b)}<p_{k m+(x+t-b+1)}<\cdots<p_{k m+k},
$$

and $x \geqslant t-1$, the pattern

$$
p_{i} p_{k m+(x-b+2)} p_{k m+(x-b+3)} \cdots p_{k m+(x+t-b)} p_{k m+(x+t-b+1)} \cdots p_{k m+x}
$$

is order-isomorphic to $q$ ( since $\left.p_{k m+(x+t-b)}<p_{i}<p_{k m+(x+t-b+1)}\right)$. Indeed, the subsequence

$$
p_{k m+(x-b+2)} p_{k m+(x-b+3)} \cdots p_{k m+(x+t-b)}
$$

is order-isomorphic to $123 \cdots(t-1), p_{i}$ comprises the $t$ term, while the subsequence

$$
p_{k m+(x+t-b+1)} p_{k m+(x+t-b+2)} \cdots p_{k m+(x-1)} p_{k m+x}
$$


is order-isomorphic to $t+1, t+2, t+3, \cdots, b$.

Thus, $p$ contains $q$, contradiction.

Lemma 4.13. For $q$ with $q_{1}=t, t \neq b$ and $b-2 \leqslant x \leqslant k-1$, we have $\left|D_{k m+x}^{k}(q)\right|=$ $\left|D_{k m+(x+1)}^{k}(q)\right|$.

Proof. We exhibit a bijection to prove the lemma. First we describe a function from $D_{k m+x}^{k}(q)$ to $D_{k m+(x+1)}^{k}(q)$. From Section 4.1, for $p \in D_{k m+x}^{k}(q)$, the injection of

$$
p_{k m+(x+t-b+2)}=p_{k m+(x+t-b+1)}+1
$$

results in a $p^{\prime} \in D_{k m+(x+1)}^{k}(q)$. We now describe the inverse function from $D_{k m+(x+1)}^{k}(q)$ to $D_{k m+x}^{k}(q)$. This direction is more straightforward. From Lemma 4.12, $\forall s \in D_{k m+(x+1)}^{k}(q)$, it follows that $p_{k m+(x+t-b+2)}=p_{k m+(x+t-b+1)}+1$. Thus, we may simply remove the value $p_{k m+(x+t-b+2)}$ from $s$ to get an $s^{\prime} \in D_{k m+x}^{k}(q)$. It is clear that the functions are inverse, and therefore bijective.

Combining Lemmata 4.11 and 4.13 yields Theorem 4.9 .

\section{The Identity Permutation}

The identity permutation merits mention. When $k=b-1$, the identity pattern has repetitions for the exact same values of $n$ as other repetitive patterns (the argument for this case is identical to the one above). For $n \geqslant k \geqslant b$ however, we have $\left|D_{n}^{k}(q)\right|=0$. Thus, only short identity patterns behave like other repetitive patterns.

\section{Implications of shape-equivalence for generalized alternating permutations}

Proposition 3.1 yields inequalities for $12 q$ and $21 q$-avoiding generalized alternating permutations. The following two theorems exploit the generality of the AD-Young diagram structure. Their proofs involve considering non-alternating AD-Young diagrams and applying the key lemmata used in the proof of Theorem 2.9.

Proposition 5.1. Let $C$ be an $r \times r$ permutation matrix. If $\mathcal{Y}=(Y, A, D)$ is an $A D$ Young diagram such that $Y$ has $n$ rows (columns) and $A \supseteq(D \cap[n-1-r])+1$, then we have

$$
\left|S_{\mathcal{Y}}\left(\left[\begin{array}{cc}
I_{2} & 0 \\
0 & C
\end{array}\right]\right)\right| \leqslant\left|S_{\mathcal{Y}}\left(\left[\begin{array}{cc}
J_{2} & 0 \\
0 & C
\end{array}\right]\right)\right| .
$$

The constraint on $A, D$ is that every required descent, except possibly those involving the last $r$ rows, must be immediately preceded by a required ascent. 
Proof. We use the notation of Section 2.1. Suppose that $N \in \mathcal{D}$, and let $f(N)=$ $\left(Y^{\prime}, A^{\prime}, D^{\prime}\right)$. We claim that if $A^{\prime}=\emptyset$, then $D^{\prime}=\emptyset$.

We prove the contrapositive; suppose that $j \in D^{\prime}$. Let $f(N)$ have $k$ rows and, for $1 \leqslant i \leqslant k$, suppose that the $i^{\text {th }}$ row of $Y^{\prime}$ was the $r_{i}^{\text {th }}$ row of $Y$ before row and column deletion. It is clear that we have $r_{j}<r_{j+1} \leqslant n-x$, and hence we have $r_{j} \leqslant n-x-1$. This yields that $r_{j}-1 \in A$, and by Proposition 2.15, we have that $j-1 \in A^{\prime}$. Taking contrapositives, we have that if $A^{\prime}=\emptyset$, then $D^{\prime}=\emptyset$. By Proposition 3.1, it follows that $\left|S_{f(N)}\left(I_{2}\right)\right| \leqslant\left|S_{f(N)}\left(J_{2}\right)\right|$ for all $N \in \mathcal{D}$.

Adding these inequalities as $N$ ranges over $\mathcal{D}$ and applying Proposition 2.16 yields that

$$
\left|S_{\mathcal{Y}}\left(\left[\begin{array}{cc}
I_{2} & 0 \\
0 & C
\end{array}\right]\right)\right|=\sum_{N \in \mathcal{T}}\left|S_{f(N)}\left(I_{2}\right)\right| \leqslant \sum_{N \in \mathcal{T}}\left|S_{f(N)}\left(J_{2}\right)\right|=\left|S_{\mathcal{Y}}\left(\left[\begin{array}{cc}
J_{2} & 0 \\
0 & C
\end{array}\right]\right)\right|,
$$

as desired.

Theorem 5.2. Suppose that $n, t$ are positive integers with $t \geqslant 2$, and $D \subseteq[n-1]$ such that $1 \notin D$ and $D \cap[n+1-t]$ does not contain any two consecutive integers. If $q$ is a permutation of $[t] \backslash[2]$, then the number of permutations of length $n$ with descent set $D$ that avoid $12 q$ is at most the number of permutations of length $n$ with descent set $D$ that avoid $21 q$.

Proof. Apply Proposition 5.1 to $\mathcal{Y}=(Y, A, D)$ with $Y=\left(n^{n}\right)$ and $A=[n-1] \backslash D$, and let $C=M(q)$.

Exchanging the roles 12 and 21 reverses the inequality sign and yields similar results.

Proposition 5.3. Let $C$ be an $r \times r$ permutation matrix. If $\mathcal{Y}=(Y, A, D)$ is an $A D$-Young diagram such that $Y$ has $n$ columns and $D \supseteq(A \cap[n-r])-1$, then we have

$$
\left|S_{\mathcal{Y}}\left(\left[\begin{array}{cc}
I_{2} & 0 \\
0 & C
\end{array}\right]\right)\right| \geqslant\left|S_{\mathcal{Y}}\left(\left[\begin{array}{cc}
J_{2} & 0 \\
0 & C
\end{array}\right]\right)\right| \text {. }
$$

Theorem 5.4. Suppose that $n, t$ are positive integers with $t>2$, and $A \subseteq[n-1]$ such that $A \cap[n+2-t]$ does not contain any two consecutive integers. If $q$ is a permutation of $[t] \backslash[2]$, then the number of permutations of length $n$ with ascent set $A$ that avoid $12 q$ is at least the number of permutations of length $n$ with ascent set $A$ that avoid $21 q$.

In particular, substituting $D=\left\{k, 2 k, \ldots, k\left\lfloor\frac{n}{k}\right\rfloor\right\}$ into Theorem 5.2 yields the inequality $\left|D_{n}^{k}(12 q)\right| \leqslant\left|D_{n}^{k}(21 q)\right|$. Similarly, substituting $A=\left\{k, 2 k, \ldots, k\left\lfloor\frac{n}{k}\right\rfloor\right\}$ into Theorem 5.4 and complementing yields that $\left|D_{n}^{k}((t+2)(t+1) w)\right| \geqslant\left|D_{n}^{k}((t+1)(t+2) w)\right|$ for all $w \in S_{t}$. It is interesting that the method that yields equalities for the $k=2$ case of alternating permutations can be generalized to yield inequalities for larger $k$. 


\section{$6 \quad$ Future directions and open problems}

The equivalence $F_{k} \underset{1-\text { SASE }}{\sim} J_{k}$, conjectured in [6, Conjecture 8.1], was recently proven by Yan [21] for $k \geqslant 3$, thereby fully extending Backelin-West-Xin's Theorem 1.1 to alternating permutations.

One can consider an analogue of AD-Young diagrams related to doubly alternating permutations by also restricting the ascent and descent sets of the transpose of a transversal. Specifically, we make the following definition.

Definition 6.1. Let $Y$ be a Young diagram with $n$ rows and columns, and suppose that $A, D, A_{2}, D_{2} \subseteq[n-1]$. We call $\mathcal{Y}=\left(Y, A, D, A_{2}, D_{2}\right)$ a double $A D$-Young diagram if $(Y, A, D)$ and $\left(Y^{t}, A_{2}, D_{2}\right)$ are AD-Young diagrams, where $Y^{t}$ denotes the transpose of $Y$.

One may then attempt to extend Yan's result that $F_{k} \underset{1-{ }_{\text {SASE }}}{\sim} J_{k}$ to the context of double AD-Young diagrams.

Furthermore, empirical data, which we provide in Section 7, suggests that most equivalences for alternating permutations are generated by the equivalence $F_{k} \underset{1-\widetilde{S A S E}_{k}}{\sim} J_{k}$ and trivial equivalences. In particular, all possible equivalences for odd-length alternating permutations among patterns of length 5 and 6 are generated in this manner, as well as all but 5 equivalences for even-length alternating permutations among patterns of length 6 . This occurrence mimics a similar phenomenon for ordinary permutations documented in [16], and "sporadic" equivalences occur between patterns of length 4.

Brute-force enumerations suggest the following conjecture, which would give Wilf-type equivalences over all descent types.

Conjecture 6.2. For all $k \geqslant 0$ and $n \geqslant 3$, we have $\left|D_{n}^{k}(2134 \cdots n)\right|=\mid D_{n}^{k}(n 123 \cdots(n-$ $1)) \mid$ as well as $\left|D_{n}^{k}(123 \cdots n(n-1))\right|=\left|D_{n}^{k}(23 \cdots n 1)\right|$.

Equally interesting are permutations that do not seem to be Wilf-equivalent to any other pattern for any descent type. For length four patterns, we have the following conjecture.

Conjecture 6.3. For all $p=1324,1342,3124,3412$ and $p \neq q \in S_{4},\left|D_{n}^{k}(p)\right| \neq\left|D_{n}^{k}(q)\right|$.

Question 6.4. Does a similar phenomenon arise for higher length patterns?

\section{Brute-Force Enumerations}

We computed $\left|A_{n}(q)\right|$ for small $n$ and short-length $q$ by brute-force computer enumeration. This data, shown in Tables 1 and 2, formed the basis of our results and conjectures.

We also computed $\left|D_{n}^{k}(q)\right|$ for small $n, k$ and short-length $q$ by similar enumerations. This data, shown in Table 3, forms the basis for our theorems of Section 4. 


\begin{tabular}{|l|c|c|c|c|c|c|}
\hline Patterns & 2 & 4 & 6 & 8 & 10 & 12 \\
\hline$(634521,652341),(534621,651342)$ & 1 & 5 & 61 & 1385 & 47860 & 2202236 \\
\hline $\begin{array}{l}(564321,654312), 645321,653421, \\
(456321,654123),(345621,651234),\end{array}$ & 1 & 5 & 61 & 1385 & 47860 & 2201540 \\
$\begin{array}{l}(234561,612345),(165432,543216), \\
(216543,432165),(126543,432156),\end{array}$ & & & & & & \\
$\begin{array}{l}321654,(213654,321465), 123456 \\
(123654,321456),(213465,213465),\end{array}$ & & & & & & \\
$(123465,213456)$ & & & & & \\
\hline $\begin{array}{l}(312654,321564),(213564,312465) \\
(123564,312456)\end{array}$ & 1 & 5 & 61 & 1385 & 47860 & 2198859 \\
\hline$(215643,431265),(125643,431256)$ & 1 & 5 & 61 & 1385 & 47860 & 2197690 \\
\hline$(214563,412365),(124563,412356)$ & 1 & 5 & 61 & 1385 & 47860 & 2197299 \\
\hline$(214653,421365),(124653,421356)$ & 1 & 5 & 61 & 1385 & 47860 & 2195798 \\
\hline$(143265,215436),(125436,143256)$ & 1 & 5 & 61 & 1344 & 44386 & 1954114 \\
\hline $\begin{array}{l}(132654,321546),(124365,214356), \\
(132465,213546),(123546,132456)\end{array}$ & 1 & 5 & 61 & 1344 & 44377 & 1951843 \\
$(124356,124356), 214365$ & & & & & \\
\hline$(564231,645312),(456231,645123)$ & 1 & 5 & 61 & 1344 & 44377 & 1951757 \\
\hline $\begin{array}{l}(564312,564312),(456312,564123), \\
(345612,561234), 456123\end{array}$ & 1 & 5 & 61 & 1344 & 44377 & 1951429 \\
\hline$(465312,564213),(456213,465123)$ & 1 & 5 & 61 & 1344 & 44342 & 1943735 \\
\hline$(215634,341265),(125634,341256)$ & 1 & 5 & 61 & 1344 & 44333 & 1940841 \\
\hline$(216534,342165),(126534,342156)$ & 1 & 5 & 61 & 1344 & 44333 & 1940623 \\
\hline$(546312,564132),(456132,546123)$ & 1 & 5 & 61 & 1344 & 44324 & 1940209 \\
\hline $\begin{array}{l}(231654,321645),(213645,231465), \\
(123645,231456)\end{array}$ & 1 & 5 & 61 & 1344 & 44306 & 1937196 \\
\hline$(216453,423165),(126453,423156)$ & 1 & 5 & 61 & 1344 & 44306 & 1936673 \\
\hline$(216345,234165),(126345,234156)$ & 1 & 5 & 61 & 1344 & 44306 & 1935009 \\
\hline$(142365,214536),(124536,142356)$ & 1 & 5 & 61 & 1344 & 44289 & 1935152 \\
\hline$(134265,215346),(125346,134256)$ & 1 & 5 & 61 & 1344 & 44289 & 1934933 \\
\hline$(214635,241365),(124635,241356)$ & 1 & 5 & 61 & 1344 & 44280 & 1932468 \\
\hline$(216435,243165),(126435,243156)$ & 1 & 5 & 61 & 1344 & 44280 & 1931424 \\
\hline$(215364,314265),(125364,314256)$ & 1 & 5 & 61 & 1344 & 44271 & 1930657 \\
\hline$(215463,413265),(125463,413256)$ & 1 & 5 & 61 & 1344 & 44271 & 1929874 \\
\hline$(216354,324165),(126354,324156)$ & 1 & 5 & 61 & 1344 & 44253 & 1926893 \\
\hline
\end{tabular}

Table 1: The size of $A_{2 n}(q)$ is given for all patterns $q \in S_{6}$ that participate in a nontrivial equivalence for even-length alternating permutations. Parentheses indicate trivial equivalences. 


\begin{tabular}{|c|c|c|c|c|c|c|c|}
\hline Patterns & 1 & 3 & 5 & 7 & 9 & 11 & 13 \\
\hline $\begin{array}{l}(654321,123456),(654312,213456), \\
(654123,321456),(651234,432156), \\
(612345,543216)\end{array}$ & 1 & 2 & 16 & 272 & 7936 & 329098 & 17316208 \\
\hline$(634521,125436),(634512,215436)$ & 1 & 2 & 16 & 272 & 7622 & 300499 & 15125692 \\
\hline$(653421,124356),(653412,214356)$ & 1 & 2 & 16 & 272 & 7622 & 300430 & 15106854 \\
\hline $\begin{array}{l}(645321,123546),(645312,213546), \\
(645123,321546)\end{array}$ & 1 & 2 & 16 & 272 & 7622 & 300430 & 15106113 \\
\hline $\begin{array}{l}(564321,123465),(456321,123654), \\
(345621,126543),(234561,165432), \\
(564312,213465),(456312,213654), \\
(345612,216543),(564123,321465), \\
(456123,321654),(561234,432165)\end{array}$ & 1 & 2 & 16 & 272 & 7622 & 300430 & 15102362 \\
\hline$(564213,312465),(456213,312654)$ & 1 & 2 & 16 & 272 & 7622 & 300172 & 15038858 \\
\hline$(435621,126534),(435612,216534)$ & 1 & 2 & 16 & 272 & 7622 & 300103 & 15012608 \\
\hline $\begin{array}{l}(465321,123564),(465312,213564), \\
(465123,321564)\end{array}$ & 1 & 2 & 16 & 272 & 7622 & 300094 & 15023874 \\
\hline$(346521,125643),(346512,215643)$ & 1 & 2 & 16 & 272 & 7622 & 300025 & 15004212 \\
\hline$(436521,125634),(436512,215634)$ & 1 & 2 & 16 & 272 & 7622 & 300025 & 14998611 \\
\hline $\begin{array}{l}(546321,123645),(546312,213645), \\
(546123,321645)\end{array}$ & 1 & 2 & 16 & 272 & 7622 & 299916 & 14987084 \\
\hline$(365421,124563),(365412,214563)$ & 1 & 2 & 16 & 272 & 7622 & 299897 & \\
\hline$(543621,126345),(543612,216345)$ & 1 & 2 & 16 & 272 & 7622 & 299768 & \\
\hline$(635421,124536),(635412,214536)$ & 1 & 2 & 16 & 272 & 7622 & 299708 & \\
\hline$(356421,124653),(356412,214653)$ & 1 & 2 & 16 & 272 & 7622 & 299698 & \\
\hline$(643521,125346),(643512,215346)$ & 1 & 2 & 16 & 272 & 7622 & 299668 & \\
\hline$(534621,126435),(534612,216435)$ & 1 & 2 & 16 & 272 & 7622 & 299658 & \\
\hline$(536421,124635),(536412,214635)$ & 1 & 2 & 16 & 272 & 7622 & 299639 & \\
\hline$(563421,124365),(563412,214365)$ & 1 & 2 & 16 & 266 & 7164 & 270463 & 13077672 \\
\hline$(564231,132465),(456231,132654)$ & 1 & 2 & 16 & 266 & 7164 & 270463 & 13077275 \\
\hline$(564132,231465),(456132,231654)$ & 1 & 2 & 16 & 266 & 7156 & 268940 & 12868164 \\
\hline$(354621,126453),(354612,216453)$ & 1 & 2 & 16 & 266 & 7156 & 268876 & \\
\hline$(463521,125364),(463512,215364)$ & 1 & 2 & 16 & 266 & 7148 & 267642 & \\
\hline$(453621,126354),(453612,216354)$ & 1 & 2 & 16 & 266 & 7148 & 267590 & \\
\hline$(364521,125463),(364512,215463)$ & 1 & 2 & 16 & 266 & 7148 & 267539 & \\
\hline
\end{tabular}

Table 2: The size of $A_{2 n+1}(q)$ is given for all patterns $q \in S_{6}$ that participate in a nontrivial equivalence for odd-length alternating permutations. Parentheses indicate trivial equivalences. 


\begin{tabular}{|l|c|c|c|c|c|c|c|c|c|}
\hline Patterns & 1 & 2 & 3 & 4 & 5 & 6 & 7 & 8 & 9 \\
\hline 1342 & 1 & 1 & 1 & 2 & 5 & 9 & 20 & 64 & 143 \\
\hline 1243 & 1 & 1 & 1 & 2 & 5 & 9 & 21 & 68 & 153 \\
\hline 1423 & 1 & 1 & 1 & 3 & 6 & 9 & 42 & 93 & 143 \\
\hline 3124 & 1 & 1 & 1 & 3 & $\mathbf{9}$ & $\mathbf{9}$ & 44 & $\mathbf{1 4 3}$ & $\mathbf{1 4 3}$ \\
\hline 2134 & 1 & 1 & 1 & 3 & $\mathbf{9}$ & $\mathbf{9}$ & 44 & $\mathbf{1 5 3}$ & $\mathbf{1 5 3}$ \\
4123 & & & & & & & & & \\
\hline
\end{tabular}

Table 3: This table shows $\left|D_{n}^{3}(q)\right|$ for selected $q \in S_{4}$. Note in particular the repeated values of $\left|D_{n}^{3}(q)\right|$ for $q=2134,4123$.

\section{Acknowledgements}

This research was done in the the MIT Math Department's PRIMES program. The authors would like to thank their mentor Dr. Joel Lewis of the University of Minnesota for his incredibly helpful guidance and insight, and for suggesting the topic of patternavoiding alternating permutations to them.

\section{References}

[1] Eric Babson and Julian West. The permutations $123 p_{4} \ldots p_{m}$ and $321 p_{4} \ldots p_{m}$ are Wilf-equivalent. Graphs and Combinatorics, 16(4):373-380, 2000.

[2] Jörgen Backelin, Julian West, and Guoce Xin. Wilf-equivalence for singleton classes. Advances in Applied Mathematics, 38(2):133-148, 2007.

[3] Sara C. Billey and Gregory S. Warrington. Kazhdan-Lusztig polynomials for 321hexagon-avoiding permutations. J. Algebraic Combinatorics, 13(2):111-136, 2001.

[4] Miklós Bóna. Combinatorics of Permutations, Second Edition. Discrete Mathematics and its Applications. CRC Press, 2012.

[5] Miklós Bóna. On a family of conjectures of Joel Lewis on alternating permutations. Graphs and Combinatorics, 2013.

[6] Nihal Gowravaram and Ravi Jagadeesan. Beyond alternating permutations: Pattern avoidance in Young diagrams and tableaux. arXiv:1301.6796v1.

[7] Ravi Jagadeesan. Ascent-descent Young diagrams and pattern avoidance in alternating permutations. Preprint.

[8] Donald E. Knuth. The Art of Computer Programming, volume 3. Addison-Wesley, 1969.

[9] Venkatraman Lakshmibai and B. Sandhya. Criterion for smoothness of Schubert varieties in $S L(n) / B$. Proc. Indian Acad. Sci. (Math. Sci.), 100(1):45-52, 1990.

[10] Joel Brewster Lewis. Pattern avoidance for alternating permutations and Young tableaux. J. Combinatorial Theory, Series A, 118(4):1436-1450, 2011. 
[11] Joel Brewster Lewis. Generating trees and pattern avoidance in alternating permutations. Electronic J. Combinatorics, 19(1):\#P21, 2012.

[12] Ian G. Macdonald. Notes on Schubert Polynomials. Publications du Laboratoire de combinatoire et d'informatique mathématique. Département de mathématiques et d'informatique, Université du Québec a Montréal, 1991.

[13] Toufik Mansour. Restricted 132-alternating permutations and Chebyshev polynomials. Annals of Combinatorics, 7(2):201-227, 2003.

[14] Eric Ouchterlony. Pattern avoiding doubly alternating permutations. In Proc. FP$S A C, 2006$. arXiv:0908.0255v1.

[15] Rodica Simion and Frank W. Schmidt. Restricted permutations. European J. Combinatorics, 6:383-407, 1985.

[16] Zvezdelina Stankova and Julian West. A new class of Wilf-equivalent permutations. J. Algebraic Combinatorics, 15:271-290, 2002.

[17] Richard P. Stanley. Catalan addendum to Enumerative Combinatorics. http://

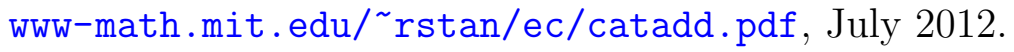

[18] Bridget Tenner. Database of permutation pattern avoidance. http://math.depaul. edu/bridget/patterns.html.

[19] Julian West. Generating trees and the Catalan and Schröder numbers. Discrete Mathematics, 146:247-262, 1995.

[20] Yuexiao Xu and Sherry H. F. Yan. Alternating permutations with restrictions and standard Young tableaux. Electronic J. Combinatorics, 19(2):\#P49, 2012.

[21] Sherry H. F. Yan. On Wilf equivalence for alternating permutations. Electronic Journal of Combinatorics, 20(3):\#P58, 2013. 